\title{
Regulating gene expression to achieve temporal precision $\star$
}

\author{
Khem Raj Ghusinga* Abhyudai Singh ** \\ * University of North Carolina at Chapel Hill, Chapel Hill, NC, USA \\ (e-mail: khem@email.unc.edu). \\ ** University of Delaware, Newark, DE, USA \\ (e-mail: absingh@udel.edu)
}

\begin{abstract}
Cellular response to an environmental change is often triggered by accumulation of an appropriate gene product up to a critical threshold. How do cells regulate gene expression to achieve precision in timing of such responses is a question of interest. Earlier work has shown that for a stable gene product, a constant rate of accumulation provides minimum noise in the time of response, provided that initial gene product distribution is degenerate. Here, we show that this strategy is no longer optimal if the initial gene product level is drawn from a non-degenerate distribution. Finally, we discuss biological relevance of these findings.
\end{abstract}

\section{INTRODUCTION}

Robust cellular function requires generating appropriate response to both internal and external cues (Kussell and Leibler, 2005; Perkins and Swain, 2009). An essential ingredient of cellular response is to accumulate a regulatory component upto a its required critical level. Such a mechanism is used for deferring decision making until sufficient evidence is gathered, or for coordinating other biological processes (Levine et al., 2012; Levine and Elowitz, 2014; Winfree, 2001; Rensing et al., 2001; Rappaport et al., 2005; Qian et al., 2019). Numerous examples of such mechanisms appear, with varying themes, in cell-fate decisions, signaling, development, apoptosis, lysis, etc. (Salz, 2007; Goldschmidt et al., 2015; McAdams and Arkin, 1997; Gupta et al., 2018; Pedraza and Paulsson, 2007; Nachman et al., 2007; Paek et al., 2016; Collart et al., 2013; Dennehy and Wang, 2011; White et al., 2011; Yurkovsky and Nachman, 2013; Chen et al., 2004; Koyama et al., 2004; Carniol et al., 2004; Piggot and Hilbert, 2004; Spencer et al., 2009; Roux et al., 2015; Kracikova et al., 2013).

It is now increasingly appreciated that gene expression is inherently a stochastic process (Cai and Xie, 2006; Raser and O'Shea, 2005; Raj and van Oudenaarden, 2008; Kærn et al., 2005; Corrigan et al., 2016; Dar et al., 2012; Hinczewski and Thirumalai, 2014). Consequently, the time at which a noisy gene product attains a critical threshold is stochastic, as shown by recent single cell experiments (Dennehy and Wang, 2011; Yurkovsky and Nachman, 2013). How is gene expression regulated to attain temporal precision, i.e., to implement precise timers, despite the inherent stochasticity is a question of interest.

To address the question of precise timers, recent works have modeled the time to attain a threshold as a firstpassage time problem (Murugan and Kreiman, 2011; Ghusinga and Singh, 2015; Lagomarsino et al., 2016; Ghusinga et al., 2017; Gupta et al., 2018). In Ghusinga et al. (2017), precision in timing was defined as the coef- ficient of variation around a given a fixed mean time. It was shown that for a stable gene product, a no feedback strategy minimizes noise in timing. This result was shown to be robust to variation in different model parameters (Ghusinga and Singh, 2017; Ghusinga et al., 2017). Moreover, Ghusinga et al. (2017) showed that for an unstable gene product, a positive feedback suppressed the noise better. Lagomarsino et al. (2016) looked at a slightly different problem of comparing the coefficient of variation of time to cross a threshold, provided that gene product reached a fixed steady-state level. In this case, the timescale of event determined the optimal feedback strategy. In the same vein, Gupta et al. (2018) examined the coefficient of variation if an upstream regulator governed the production of the gene product of interest and showed that such a regulation can make the timing more precise.

One assumption these works make is that the gene product level is deterministically equal to zero at $t=0$, which corresponds to the time when stimulus arrives and initiates accumulation of the gene product. This assumption may be violated in some cases. For example, consider a scenario in which the gene product is repressed, until stimulus derepresses it to allow its accumulation. However, if the repression is not perfect, then there may be some leaky expression, resulting in a non-zero amount of gene product at $t=0$. In this work, we explore whether the no feedback strategy remains to be the best strategy to minimize noise in timing around a fixed mean time, if the initial gene product level is drawn from a non-degenerate distribution. We show that this is not the case, and the optimal feedback strategy depends upon the initial distribution.

We begin by summarizing the previous results on firstpassage time calculations and extend them for the case when initial gene product is drawn from a distribution. Then, we obtain results on optimal feedback strategy, and conclude the paper by discussing biological relevance of the results.

\footnotetext{
* AS is supported by NIH 1R01GM124446-01.
} 


\section{PRELIMINARIES}

In this section, we describe a model of gene product accumulation and compute its first-passage time distribution. The derivations largely follow Ghusinga et al. (2017).

\subsection{Setup}

Let $x(t)$ denote the level of a regulatory gene product (protein) at time $t$ (Fig. 1). Depending upon the required complexity, the time evolution of $x(t)$ may include the dynamics of gene (e.g., switching between on and off states), transcription (mRNA synthesis from a gene), translation (protein synthesis from an mRNA), mRNA degradation, and protein degradation Paulsson (2005). For analytical tractability, here we consider a rather simple case wherein protein is produced in geometrically distributed bursts. This model corresponds to the scenario wherein the gene is constitutively active, and the mRNA half life is much smaller than that of the protein (Paulsson, 2005; Elgart et al., 2011; Shahrezaei and Swain, 2008).

The model can be specified by the probabilities of protein production and degradation events taking place in an infinitesimal time interval $(t, t+d t)$ as

$$
\begin{aligned}
& \text { Probability }(x(t)=i+B \mid x(t)=i)=k_{i} d t \\
& \operatorname{Probability}(x(t)=i-1 \mid x(t)=i)=i \gamma d t, \\
& \operatorname{Probability}(B=i)=\frac{b^{i}}{(b+1)^{i+1}}, b \geq 0, i \in\{0,1,2, \ldots\}
\end{aligned}
$$

Here $k_{i}: \mathbb{R}_{+} \rightarrow \mathbb{R}_{+}$represents the protein production rate when the protein level is $x(t)=i$. It is taken to be an increasing function in its argument if the protein production is regulated via a positive feedback. Likewise, $k_{i}$ being decreasing function implements a negative feedback and $k_{i}$ being constant represents a no feedback mechanism. The parameter $\gamma$ denotes the degradation rate of one protein molecule. Furthermore, $B$ denotes the burst size which follows a geometric distribution with mean $b$.

\subsection{First-passage time}

We are interested in studying timers implemented via this protein. We say that an event of interest is triggered by if it occurs when $x(t) \geq X$ for the first time (Fig. 1). Formally, the triggering time of a timer can be described as

$$
T:=\inf \{t \geq 0: x(t) \geq X \mid x(0)<X\} .
$$

The probability density function (pdf) of the first-passage time (FPT), $T$, is computed by constructing an auxiliary process that has same probabilities of occurrences as $x(t)$ in (1)-(3) except that the auxiliary process is absorbed as soon as the protein count crosses $X$. Following Ghusinga et al. (2017), the pdf of $T$ is computed by

$$
f_{T}(t)=\boldsymbol{U}^{\top} \exp (\boldsymbol{A} t) \boldsymbol{P}(0),
$$

where $\boldsymbol{U}$ represents the following $X$-dimensional vector

$$
\boldsymbol{U}=\left[k_{0}\left(\frac{b}{b+1}\right)^{X} k_{1}\left(\frac{b}{b+1}\right)^{X-1} \cdots k_{X-1} \frac{b}{b+1}\right]^{\top}
$$
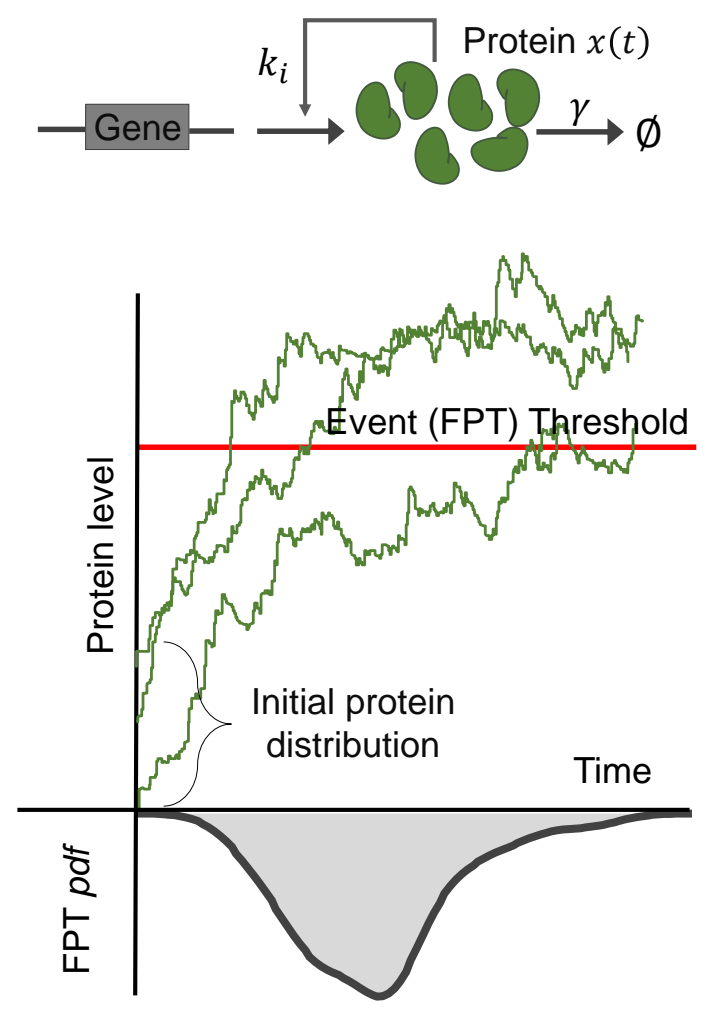

Fig. 1. Setting up first-passage time problem for gene expression model. Top: Gne expression model that includes production and degradation of protein, $x(t)$. The production is assumed to occur in bursts. Bottom: Timer is triggered when $x(t)$ reaches a critical level for the first time. Each protein trajectory represents protein level over time inside individual cells. The initial protein level is drawn from a nondegenerate distribution.

and $\boldsymbol{A}$ is an $X \times X$ matrix with elements

$$
a_{i j}=\left\{\begin{array}{ll}
0, & j>i+1 \\
(i-1) \gamma, & j=i+1 \\
-k_{i-1} \frac{b}{b+1}-(i-1) \gamma, & j=i \\
k_{i-1} \frac{b^{i-j}}{(b+1)^{i-j+1}}, & j<i
\end{array} .\right.
$$

Finally, $\boldsymbol{P}(0)$ is an $X$-dimensional vector representing the distribution of protein level at $t=0$. We can represent $\boldsymbol{P}(0)$ by

$$
\boldsymbol{P}(0)=\left[\begin{array}{lllll}
q_{0} & q_{1} & \cdots & q_{X-2} & q_{X-1}
\end{array}\right]^{\top},
$$

where $\sum_{i=0}^{X-1} q_{i}=1$ and $q_{i}>0 \forall i \in[0, X-1]$. In Ghusinga et al. (2017), we considered a degenerate distribution $\boldsymbol{P}(0)$, where $q_{0}=1$.

The matrix $\boldsymbol{A}$ satisfies following properties that would be useful in our analysis.

(1) $\boldsymbol{A}$ is a Hurwitz matrix. That is, it is invertible and has eigenvalues that have negative real parts.

(2) For $\gamma=0, \boldsymbol{A}^{-1}$ is given by 


$$
\boldsymbol{A}^{-1}=-\frac{1}{b}\left[\begin{array}{ccccc}
\frac{b+1}{k_{0}} & 0 & \cdots & 0 & 0 \\
\frac{1}{k_{1}} & \frac{b+1}{k_{1}} & \cdots & 0 & 0 \\
\frac{1}{k_{2}} & \frac{1}{k_{2}} & \cdots & 0 & 0 \\
\vdots & \vdots & \ddots & \vdots & \vdots \\
\frac{1}{k_{X-2}} & \frac{1}{k_{X-2}} & \cdots & \frac{b+1}{k_{X-2}} & 0 \\
\frac{1}{k_{X-1}} & \frac{1}{k_{X-1}} & \cdots & \frac{1}{k_{X-1}} & \frac{b+1}{k_{X-1}}
\end{array}\right] .
$$

When a non-bursty production is considered, then $\boldsymbol{A}^{-1}$ simplifies to

$$
\boldsymbol{A}^{-1}=-\left[\begin{array}{ccccc}
\frac{1}{k_{0}} & 0 & \cdots & 0 & 0 \\
\frac{1}{k_{1}} & \frac{1}{k_{1}} & \cdots & 0 & 0 \\
\frac{1}{k_{2}} & \frac{1}{k_{2}} & \cdots & 0 & 0 \\
\vdots & \vdots & \ddots & \vdots & \vdots \\
\frac{1}{k_{X-2}} & \frac{1}{k_{X-2}} & \cdots & \frac{1}{k_{X-2}} & 0 \\
\frac{1}{k_{X-1}} & \frac{1}{k_{X-1}} & \cdots & \frac{1}{k_{X-1}} & \frac{1}{k_{X-1}}
\end{array}\right] .
$$

(3) $\boldsymbol{U}^{\top} \boldsymbol{A}^{-1}=-\left[\begin{array}{llll}1 & 1 & \ldots & 1\end{array}\right]$.

We do not provide proofs for these properties and refer the interested reader to Ghusinga et al. (2017).

Exploiting the first property above, the probability density function in (5) can be further used to compute moments of the first-passage time, $T$, as

$$
\begin{aligned}
\left\langle T^{m}\right\rangle=(-1)^{m+1} m ! \boldsymbol{U}^{\top}\left(\boldsymbol{A}^{-1}\right)^{m+1} \boldsymbol{P}(0), \\
m=\{0,1,2, \ldots\} .
\end{aligned}
$$

The properties (2) and (3) of $\boldsymbol{A}$ are useful in simplifying the computation of moments given by above formula. With these formulas, we can now compute the optimal feedback strategy that would minimize the noise in timing around a fixed mean.

\section{OPTIMAL FEEDBACK STRATEGY WHEN INITIAL PROTEIN LEVEL IS RANDOM}

To determine the optimal feedback strategy, we begin by computing the first two moments of $T$. To this end, we utilize the properties (2) and (3) from previous section.

To compute $\langle T\rangle$, we have that

$$
\langle T\rangle=\boldsymbol{U}^{\top} \boldsymbol{A}^{-1} \boldsymbol{A}^{-1} \boldsymbol{P}(0) .
$$

By property (3), we already have $\boldsymbol{U}^{\top} \boldsymbol{A}^{-1}$. Thus, we only need to compute $\boldsymbol{A}^{-1} \boldsymbol{P}(0)$. Using property (2), we have

$$
\langle T\rangle=\frac{1}{b} \sum_{i=0}^{X-1} \frac{\sum_{l=0}^{i} q_{l}}{k_{i}}+\sum_{i=0}^{X-1} \frac{q_{i}}{k_{i}} .
$$

If we consider the non-bursty production, we can use the corresponding $\boldsymbol{A}^{-1}$ from property (2) and get

$$
\langle T\rangle=\sum_{i=0}^{X-1} \frac{\sum_{l=0}^{i} q_{l}}{k_{i}} .
$$

The second order moment $\left\langle T^{2}\right\rangle$ can be computed in the same fashion. In particular, we obtain

$$
\begin{array}{r}
\left\langle T^{2}\right\rangle=\frac{2}{b^{2}} \sum_{i=0}^{X-1} \frac{\sum_{l=0}^{i} q_{l}}{k_{i}}\left(\sum_{j=i}^{X-1} \frac{1}{k_{j}}\right)+\frac{2}{b} \sum_{i=0}^{X-1} \frac{\sum_{l=0}^{i} q_{l}}{k_{i}^{2}} \\
+\frac{2}{b} \sum_{i=0}^{X-1} \frac{q_{i}}{k_{i}}\left(\sum_{j=i}^{X-1} \frac{1}{k_{j}}\right)+2 \sum_{i=0}^{X-1} \frac{q_{i}}{k_{i}^{2}}
\end{array}
$$

The above formula simplifies a bit if we consider nonbursty production instead

$$
\left\langle T^{2}\right\rangle=2 \sum_{i=0}^{X-1} \frac{\sum_{l=0}^{i} q_{l}}{k_{i}}\left(\sum_{j=i}^{X-1} \frac{1}{k_{j}}\right) .
$$

As a sanity check, the formulas for first two moments, both for bursty and non-bursty production, should reduce to those obtained by Ghusinga et al. (2017) when the initial protein distribution is considered as $x(0)=0$ with probability one. Indeed, setting $q_{0}=1$ and $q_{i}=0$ for $i>0$ results in the following moments for the bursty production case

$$
\begin{aligned}
\langle T\rangle= & \frac{1}{b} \sum_{i=0}^{X-1} \frac{1}{k_{i}}+\frac{1}{k_{0}} \\
\left\langle T^{2}\right\rangle= & \frac{2}{b^{2}} \sum_{i=0}^{X-1} \frac{1}{k_{i}}\left(\sum_{j=i}^{X-1} \frac{1}{k_{j}}\right)+\frac{2}{b} \sum_{i=0}^{X-1} \frac{1}{k_{i}^{2}} \\
& +\frac{2}{b} \frac{1}{k_{0}^{2}} \sum_{j=0}^{X-1} \frac{1}{k_{j}}+\frac{2}{k_{0}^{2}},
\end{aligned}
$$

which is consistent with prior results. Likewise, we can show that the results are consistent with prior results for the special case of non-bursty production.

Having derived the expressions for the first two moments, we can now set up the problem of finding optimal feedback strategy. We set up the an optimization problem as

$$
\min _{k_{0}, k_{1}, \ldots, k_{X-1}, \lambda}\left\langle T^{2}\right\rangle+\lambda(\langle T\rangle-c),
$$

where $c$ is the given fixed mean time and $\lambda$ is Lagrange multiplier (Ghusinga et al., 2017). While it is possible to numerically solve the problem for both bursty and nonbursty production, it is less complicated to examine it for the non-bursty production.

Let us use a shorthand notation $w_{i}=\sum_{l=0} i q_{i}$. Then, we have that for non-bursty production

$$
\begin{aligned}
\frac{d\langle T\rangle}{d k_{i}} & =-\frac{w_{i}}{k_{i}^{2}} \\
\frac{d\left\langle T^{2}\right\rangle}{d k_{i}} & =-\frac{1}{k_{i}^{2}}\left(\sum_{j=0}^{i-1} \frac{w_{j}}{k_{j}}+\frac{2 w_{i}}{k_{i}}+w_{i} \sum_{j=i+1}^{X-1} \frac{1}{k_{j}}\right)
\end{aligned}
$$

Using these, the fixed points of the objective function (19) are computed by setting its derivatives with respect to the optimization variables $k_{0}, \ldots, k_{X-1}, \lambda$ equal to zero. This results in 


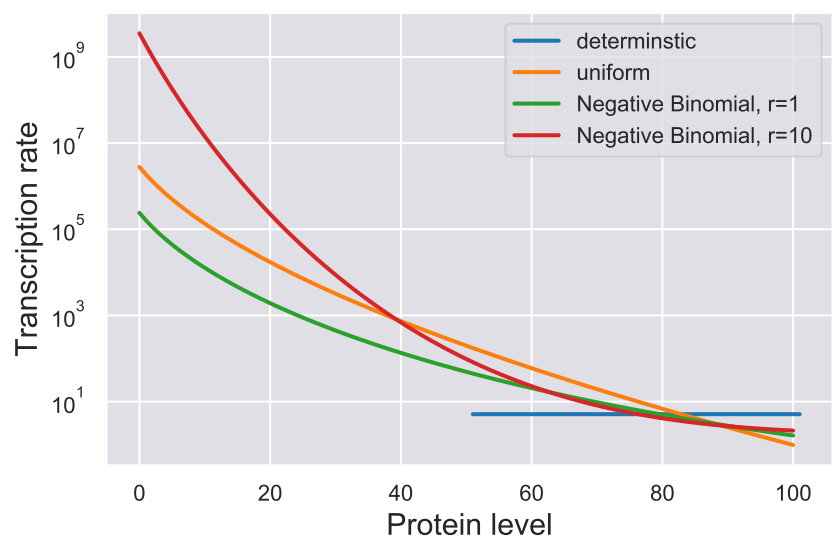

Fig. 2. Optimal production rates for non-bursty production for various distributions of initial protein level. The parameters are chosen as $X=50, c=10$. The distributions are truncated to the finite support $[0, X-1]$ and their parameters are chosen such that the initial mean protein level is $\sim X / 2$. For Negative Binomial, the additional free parameter for its shape is chosen as shown.

$$
\begin{aligned}
& \sum_{j=0}^{i-1} \frac{w_{j}}{k_{j}}+\frac{2 w_{i}}{k_{i}}+w_{i} \sum_{j=i+1}^{X-1} \frac{1}{k_{j}}+w_{i} \lambda=0 \\
& \sum_{i=0}^{X-1} \frac{w_{i}}{k_{i}}-c=0
\end{aligned}
$$

These equations form a linear system that can be solved using off-the-shelf numerical programs.

In Fig. 2, we plot the optimal rates $k_{i}$ for various distributions for initial protein level at $t=0$. For a given event threshold $X$, we set an average protein count approximately $X / 2$ and consider different distributions of protein level on the support $[0, X-1]$. As a limiting case, we consider the de-generate distribution, which indeed shows that the solution to the above linear system is given by a no feedback strategy, where $k_{i}=X / c$. As a hypothetical case, we consider uniformly distributed protein count over the interval. Here, the optimal feedback strategy is a negative feedback: a very high transcription rate for low protein counts, which decreases as protein level increases. For a biologically relevant case, we considered a Negative Binomial distribution, which not only corresponds to steady-state distribution of protein level in for some models Paulsson (2005), but also is a limiting case of Geometric and Poisson distributions. We considered Negative Binomial with shape parameter $r$ and probability parameter $p$ which is set to get a mean of initial distribution $\sim X / 2$. Even for this distribution, a negative feedback is optimal.

\section{CONCLUSION}

In this paper, we investigated how gene expression can be regulated to ensure precision in the timer that triggers upon attainment of a critical threshold. Previously, this problem has been studied for a case where initial protein distribution is deterministic. Here we relaxed this assumption and drew the initial protein level from a discrete distribution over finite support. Our results show that whereas for the deterministic initial condition, a constant production rate results in most precise timer, a negative feedback on the production rate is required if the initial condition is drawn from a distribution. These results underscore the utility of negative feedback in genetic circuits. In future work, it would be interesting to explore the optimal feedback strategies when the protein degrades.

\section{REFERENCES}

Cai, L. and Xie, N.F.X.S. (2006). Stochastic protein expression in individual cells at the single molecule level. Nature, 440, 358-362.

Carniol, K., Eichenberger, P., and Losick, R. (2004). A threshold mechanism governing activation of the developmental regulatory protein $\sigma \mathrm{f}$ in bacillus subtilis. Journal of Biological Chemistry, 279, 14860-14870.

Chen, K.C., Calzone, L., Csikasz-Nagy, A., Cross, F.R., Novak, B., and Tyson, J.J. (2004). Integrative analysis of cell cycle control in budding yeast. Molecular Biology of the Cell, 15, 3841-3862.

Collart, C., Allen, G.E., Bradshaw, C.R., Smith, J.C., and Zegerman, P. (2013). Titration of four replication factors is essential for the Xenopus laevis midblastula transition. Science, 341, 893-896.

Corrigan, A.M., Tunnacliffe, E., Cannon, D., and Chubb, J.R. (2016). A continuum model of transcriptional bursting. eLife, 5, e13051.

Dar, R.D., Razooky, B.S., Singh, A., Trimeloni, T.V., McCollum, J.M., Cox, C.D., Simpson, M.L., and Weinberger, L.S. (2012). Transcriptional burst frequency and burst size are equally modulated across the human genome. Proceedings of the National Academy of Sciences, 109, 17454-17459.

Dennehy, J.J. and Wang, I.N. (2011). Factors influencing lysis time stochasticity in bacteriophage $\lambda$. $B M C$ Microbiology, 11, 174.

Elgart, V., Jia, T., Fenley, A.T., and Kulkarni, R. (2011). Connecting protein and mrna burst distributions for stochastic models of gene expression. Physical Biology, 8, 046001.

Ghusinga, K.R., Dennehy, J.J., and Singh, A. (2017). First-passage time approach to controlling noise in the timing of intracellular events. Proceedings of the $\mathrm{Na}$ tional Academy of Sciences, 114, 693-698.

Ghusinga, K.R. and Singh, A. (2015). Theoretical predictions on the first-passage time for a gene expression model. In 2015 54th IEEE Conference on Decision and Control (CDC), 3864-3869. IEEE.

Ghusinga, K.R. and Singh, A. (2017). Effect of geneexpression bursts on stochastic timing of cellular events. In 2017 American Control Conference (ACC), 21182123. IEEE.

Goldschmidt, Y., Yurkovsky, E., Reif, A., Rosner, R., Akiva, A., and Nachman, I. (2015). Control of relative timing and stoichiometry by a master regulator. PLOS ONE, 10, 1-14.

Gupta, S., Varennes, J., Korswagen, H.C., and Mugler, A. (2018). Temporal precision of regulated gene expression. PLoS computational biology, 14, e1006201.

Hinczewski, M. and Thirumalai, D. (2014). Cellular signaling networks function as generalized wiener-kolmogorov filters to suppress noise. Physical Review X, 4, 041017. 
Kærn, M., Elston, T.C., Blake, W.J., and Collins, J.J. (2005). Stochasticity in gene expression: from theories to phenotypes. Nature Reviews Genetics, 6, 451-464.

Koyama, T., Iwami, M., and Sakurai, S. (2004). Ecdysteroid control of cell cycle and cellular commitment in insect wing imaginal discs. Molecular and Cellular Endocrinology, 213, 155-166.

Kracikova, M., Akiri, G., George, A., Sachidanandam, R., and Aaronson, S. (2013). A threshold mechanism mediates p53 cell fate decision between growth arrest and apoptosis. Cell Death \& Differentiation, 20, 576588 .

Kussell, E. and Leibler, S. (2005). Phenotypic diversity, population growth, and information in fluctuating environments. Science, 309, 2075-2078.

Lagomarsino, M.C., Caselle, M., Osella, M., et al. (2016). Stochastic timing in gene expression for simple regulatory strategies. Nucleic Acids Research, gkw1235.

Levine, J.H. and Elowitz, M.B. (2014). Polyphasic feedback enables tunable cellular timers. Current Biology, (20), R994-R995.

Levine, J.H., Fontes, M.E., Dworkin, J., and Elowitz, M.B. (2012). Pulsed feedback defers cellular differentiation. PLoS Biol, 10, e1001252.

McAdams, H.H. and Arkin, A.P. (1997). Stochastic mechanisms in gene expression. Proceedings of the National Academy of Sciences, 94, 814-819.

Murugan, R. and Kreiman, G. (2011). On the minimization of fluctuations in the response times of autoregulatory gene networks. Biophysical journal, 101, 1297-1306.

Nachman, I., Regev, A., and Ramanathan, S. (2007). Dissecting timing variability in yeast meiosis. Cell, 131, 544-556.

Paek, A.L., Liu, J.C., Loewer, A., Forrester, W.C., and Lahav, G. (2016). Cell-to-cell variation in p53 dynamics leads to fractional killing. Cell, 165(3), 631-642.

Paulsson, J. (2005). Model of stochastic gene expression. Physics of Life Reviews, 2, 157-175.

Pedraza, J.M. and Paulsson, J. (2007). Random timing in signaling cascades. Molecular Systems Biology, 3, 81 .

Perkins, T.J. and Swain, P.S. (2009). Strategies for cellular decision-making. Molecular systems biology, 5, 326.

Piggot, P.J. and Hilbert, D.W. (2004). Sporulation of bacillus subtilis. Current Opinion in Microbiology, 7,
$579-586$.

Qian, J., Gelens, L., and Bollen, M. (2019). Coordination of timers and sensors in cell signaling. BioEssays, 41(3), 1800217.

Raj, A. and van Oudenaarden, A. (2008). Nature, nurture, or chance: stochastic gene expression and its consequences. Cell, 135, 216-226.

Rappaport, N., Winter, S., and Barkai, N. (2005). The ups and downs of biological timers. Theoretical Biology and Medical Modelling, 2, 22.

Raser, J.M. and O'Shea, E.K. (2005). Noise in gene expression: Origins, consequences, and control. Science, 309, $2010-2013$.

Rensing, L., Meyer-Grahle, U., and Ruoff, P. (2001). Biological timing and the clock metaphor: oscillatory and hourglass mechanisms. Chronobiology international, 18, 329-369.

Roux, J., Hafner, M., Bandara, S., Sims, J.J., Hudson, H., Chai, D., and Sorger, P.K. (2015). Fractional killing arises from cell-to-cell variability in overcoming a caspase activity threshold. Molecular Systems Biology, $11,803$.

Salz, H. (2007). Male or female? The answer depends on when you ask. PLOS Biology, 5, e335.

Shahrezaei, V. and Swain, P.S. (2008). Analytical distributions for stochastic gene expression. Proceedings of the National Academy of Sciences, 105, 17256-17261.

Spencer, S.L., Gaudet, S., Albeck, J.G., Burke, J.M., and Sorger, P.K. (2009). Non-genetic origins of cell-to-cell variability in TRAIL-induced apoptosis. Nature, 459, 428-432.

White, R., Chiba, S., Pang, T., Dewey, J.S., Savva, C.G., Holzenburg, A., Pogliano, K., and Young, R. (2011). Holin triggering in real time. Proceedings of the National Academy of Sciences, 108, 798-803.

Winfree, A.T. (2001). The geometry of biological time, volume 12. Springer Science \& Business Media.

Yurkovsky, E. and Nachman, I. (2013). Event timing at the single-cell level. Briefings in Functional Genomics, $12,90-98$. 